\title{
Disfunção temporomandibular e o impacto na qualidade de vida:
} uma revisão de literatura

\section{Temporomandibular dysfunction and the impact on quality of life: a literature review}

\author{
Letícia Alves Novaes', Tamires de Sá Barreto Dantas², Viviane Maria Gonçalves de Figueiredo³ \\ 'Graduanda em Odontologia no Centro Universitário Leão Sampaio. Juazeiro do Norte, Ceará, Brasil. carol_lethy@hotmail.com \\ ${ }^{2}$ Graduanda em Odontologia no Centro Universitário Leão Sampaio. Juazeiro do Norte, Ceará, Brasil. tamires_dantas01@Hotmail.com \\ ${ }^{3}$ Autora para correspondência. Doutora em Odontologia Restauradado. Docente no Centro Universitário Leão Sampaio. \\ Juazeiro do Norte, Ceará, Brasil.vivi_mfigueiredo@yahoo.com.br
}

Resumo | A disfunção temporomandibular tem apresentando-se frequente na população, assim dor crônica está diretamente relacionada com o estado da qualidade de vida do indivíduo; por isso se faz necessário compreender a interferência na qualidade de vida de indivíduos acometidos pela disfunção. Objetivou-se revisar a literatura sobre disfunção temporomandibular e a qualidade de vida dos portadores da mesma, enfatizando os diversos índices para avaliar a qualidade de vida. As bases de dados para busca da literatura foram Pubmed, Bireme, Lilacs, Scielo. As palavras-chaves usadas na busca da literatura foram Epidemiologia, Transtornos da Articulação Temporomandibular, Qualidade de Vida, presentes no Desc Saúde. A literatura selecionada para esta revisão foi selecionada com base nos resumos e abstracts. Os critérios de inclusão da literatura nesta seleção foram Estudos Epidemiológicos e Clínicos; Revisão Sistemática e Metanálise; Literatura em Português e Inglês; Estudos que avaliem a DTM e Índices de Qualidade de Vida; enquanto que os critérios de exclusão foram Revisão de Literatura, Caso Clínico, Carta ao Editor; Literatura que abordasse a DTM e Qualidade de Vida em Crianças; Estudos que não abordassem as variáveis em estudo. A disfunção temporomandibular conforme a literatura revisada apresenta impacto na qualidade de vida do indivíduo portador da disfunção, diversos são os questionários para mensurar o quanto a DTM interfere nas atividades diárias dos pacientes, no entanto a associação de instrumentos é favorável para avaliar diferentes categorias quanto a qualidade de vida dos pacientes com disfunção.

Palavras-chave: Epidemiologia, Transtornos da Articulação Temporomandibular, Qualidade de Vida.

\begin{abstract}
I Temporomandibular dysfunction has been frequent in the population, so chronic pain is directly related to the state of the quality of life; so it is necessary to understand the interference in the quality of life of individuals with the dysfunction. The objective of this study was to review the literature about quality of life of patients with temporomandibular dysfunction, emphasizing the different indexes to evaluate the quality of life. The databases for searching the literature were Pubmed, Bireme, Lilacs, Scielo. The keywords used in the literature search were epidemiology, temporomandibular joint disorders, quality of life, present in Health Desc. The literature reviewed was selected based on abstracts. The inclusion criteria were epidemiological and clinical studies; systematic review and meta-analysis; literature in portuguese and english; studies evaluating the TMD and quality of life indexes; while the exclusion criteria were literature review, clinical case, letter to the editor; literature addressing TMD and quality of life in children; studies that did not address the variables under study. The temporomandibular dysfunction according to the reviewed literature has an impact on the quality of life of the individual with dysfunction, several are the questionnaires to measure how much the TMD interferes in the daily activities of the patients, nevertheless the association of instruments is favorable to evaluate different categories as to quality of life of patients with dysfunction.
\end{abstract}

Keyword: Epidemiology, Temporomandibular Joint Disorders, Quality of Life. 


\section{Introdução}

A disfunção temporomandibular (DTM) pode ser desencadeada por diversos fatores, como alterações ósseas, fatores psicológicos, fatores emocionais, perdas dentais, parafunções, postura, podendo estes também estarem associados'. $O$ diagnóstico para a DTM deve ser diferenciado, devendo ser avaliado diversos fatores, em que esse diagnóstico é estabelecido por meio de questionários, os quais identificam a queixa principal, a exatidão do local da dor e avalia a condição psicossocial do paciente ${ }^{2,3,4}$.

A disfunção temporomandibular e a dor orofacial, por ser cada dia mais frequente, vêm trazendo prejuízo para a população. A dor crônica está diretamente relacionada com o estado da qualidade de vida do indivíduo, pois as mesmas geram efeitos não só ao corpo, como também ao estado psicológico e a convivência social, que devem ser avaliadas com atenção, e que, na maioria das vezes, necessitam de tratamentos terapêuticos e multiprofissional para controlar ou reverter o caso ${ }^{5}$.

Ainda que existam indícios para crer que a DTM e outas alterações dolorosas da face causem algum impacto na qualidade de vida, poucos estudos documentam a utilização de questionários específicos ou ferramentas multidimensionais para esse fim ${ }^{6}$.

Devido a DTM ser tão prevalente na população, se faz necessário compreender se esta disfunção interfere na qualidade de vida dos portadores; objetiva-se revisar a literatura sobre disfunção temporomandibular e a qualidade de vida dos portadores da mesma, enfatizando os diversos índices para avaliar a qualidade de vida.

\section{Metodologia}

Esta revisão de literatura utilizou como base de dados: Pudmed - https://www.ncbi.nlm.nih.gov/ pubmed; Bireme - http://bvsalud.org/; Lilacs http://lilacs.bvsalud.org/ e Scielo - http://www. scielo.org/php/index.php. Foram selecionadas 44 literaturas, sendo que o total de achados foi de 406 literaturas.

As palavras-chaves usadas na busca da literatura foram Epidemiologia, Transtornos da Articulação Temporomandibular, Qualidade de Vida, presentes no Desc Saúde. A literatura selecionada para esta revisão foi selecionada com base nos resumos e abstracts. Os critérios de inclusão e exclusão da literatura estão dispostos no QUADRO 1.

Quadro 1. Critérios de Inclusão e Exclusão da literatura.

\begin{tabular}{|l|l|}
\hline \multicolumn{1}{|c|}{ Critérios de Inclusão } & \multicolumn{1}{c|}{ Critérios de Exclusão } \\
\hline Estudos Epidemiológicos e Clínicos; & Revisão de Literatura, Caso Clínico, Carta ao \\
Revisão Sistemática e Metanálise; & Editor; \\
Literatura em Português e Inglês; & Literatura que abordasse a DTM e Qualidade \\
Estudos que avaliem a DTM e Î́ndices de & de Vida em Crianças; \\
Qualidade de Vida. & $\begin{array}{l}\text { Estudos que não abordassem as variáveis em } \\
\text { estudo. }\end{array}$ \\
\hline
\end{tabular}

\section{Revisão de liłeratura}

Na busca da literatura não foi estipulado um critério de inclusão cronológico, pois observou-se que os estudos quanto a qualidade de vida de pacientes portadores de DTM se faz mais prevalente a partir dos anos 2000, e com base no GRÁFICO 1 a frequência pesquisas científicas ainda é discreta. Por isso que foram considerados os estudos significativos, em diferentes populações e culturas. 


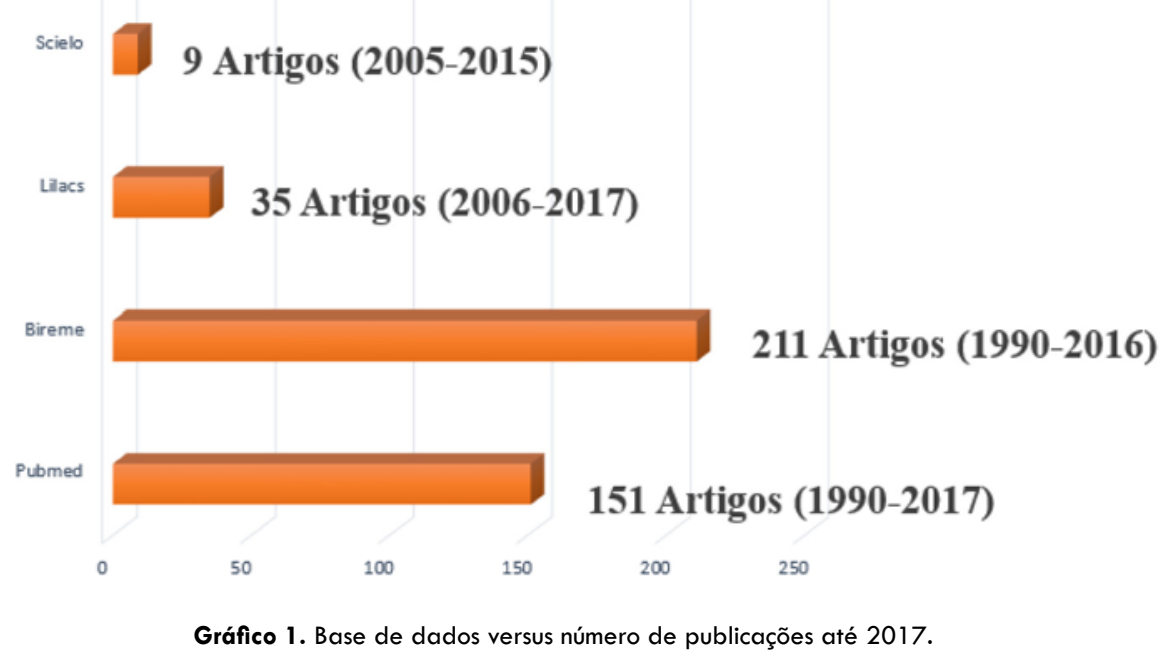

\section{Disfunção Temporomandibular}

A disfunção temporomandibular (DTM) descrita como um grupo de alterações clínicas de má funcionamento mandibular, podendo vir junto ou não de dor, que podem ser produzidas por fatores agressores à formação ou função do sistema estomatognático ${ }^{7}$. Tais disfunções incluem uma série de problemas clínicos que envolvem a musculatura mastigatória, a articulação temporomandibular (ATM) ou ambos, sendo que tais alterações interefrem na qualidade do indivíduo portador da mesma ${ }^{8}$. Tomaz-Morais et al. ${ }^{9}$ define a DTM como um termo clínico utilizado para definir um grupo de alterações na articulação temporomandibular (ATM), na musculatura da mastigação, e estruturas faciais associadas, onde essas alterações musculares e esqueléticas são consideradas os fatores desencadeantes de dor não dental na região orofacial.

Esta disfunção atinge tanto homens, quanto mulheres de todas as idades ${ }^{2,10}$. Entre os diversos sinais e sintomas, os principais são dor na região orofacial, dificuldade de movimentar a mandíbula, ruído ou estalido na articulação, e sensibilidade na musculatura'111-14. As alterações da DTM desencadeiam dor na musculatura da face, deslocam - disco articular, provocam dor na articulação e alteração da função, podendo causar inflamação. Promovendo assim limitações físicas e funcionais aos portadores $^{15}$. Dentre os diversos sinais e sintomas apresentados pela DTM a dor é a que mais interfere nas atividades diárias do indivíduo, ocasionando um efeito negativo na vida social, emocional, e nível energético da pessoa afetada ${ }^{14}$.
Por a DTM tratar de sintomatologias diversas, torna o diagnóstico e o tratamento mais complexo, envolvendo episódios de dor e movimentação muscular involuntária, que relacionam o desequilíbrio biomecânico não somente das articulações como também da região cervical ${ }^{14}$. Assim os pacientes com DTM necessitam de múltiplos focos de atenção, indicadores psicológicos, de saúde geral e qualidade de vida ${ }^{15}$.

\section{Etiologia DTM}

A etiologia da disfunção temporomandibular (DTM) caracteriza-se por seu multifatorialismo, estando associada a fatores dentais e faciais, pois não existe um único fator que desencadei todos os sinais e sintomas ${ }^{2,16,4,16}$.

Sendo proposto o entendimento de um contexto biopsicossocial da disfunção. Os fatores etiológicos são classificados pela capacidade de se iniciar e permanecer em uma condição especifica. $O$ conhecimento das manifestações que ocasiona e os fatores associados são essenciais para aprimorar as medidas preventivas $^{18}$.

De acordo com os princípios etiológicos atuais, as condições sistêmicas físicas e psicológicas são elementos responsáveis pelas alterações decorrentes na DTM. O indivíduo passa por variações comportamentais como o estresse e a ansiedade que são capazes de causar alterações dos impulsos nociceptivos do sistema nervoso central e a liberação de neurotransmissores ${ }^{15}$. 
As alterações psicológicas influenciam na repetição, intensidade e durabilidade dos hábitos parafuncionais, que são caracterizados por apertamento dos dentes e bruxismo que ocasiona hiperatividade dos músculos da mastigação. A DTM é considerada como um fator negativo sobre a saúde sistêmica e qualidade de vida, sendo caracterizada pela dor e o estresse, fatores que influenciam e comprometem as atividades diárias na escola ou trabalho, nas funções sociais, no equilíbrio afetivo e cognitivo, no sono e atividades físicas ${ }^{15}$.

Os hábitos parafuncionais são vistos como fatores de ameaça para a DTM, conforme Poveda et al. ${ }^{19}$ (2007), sendo definidos como quaisquer hábitos que fujam da normalidade e ocasione danos a um órgão ou sistema. Entre essas práticas estão mascar chiclete, morder lápis, roer unhas, ranger ou apertar dentes, onde o indivíduo encontra nesse vício uma maneira de liberar a tensão emocional ${ }^{3}$. Estes hábitos levam ao comprometimento de fatores fisiológicos ou anatômicos do sistema estomatognático, possibilitando alterações aos ossos, dentes e tecidos moles ${ }^{3}$.

A abordagem multidisciplinar para minimizar a dor deve ser considerada para o tratamento destas alterações incluindo modalidades nãofarmacológicas e farmacológicas ${ }^{8}$. Bem como, pacientes com diagnóstico de desordem muscular apresetam risco para desenvolver a cronicidade do problema e apresentam um difícil controle dos sinais e sintomas ${ }^{20}$. A FIGURA 1 representa um esquema sobre os fatores etiológicos e perpertuantes da DTM.

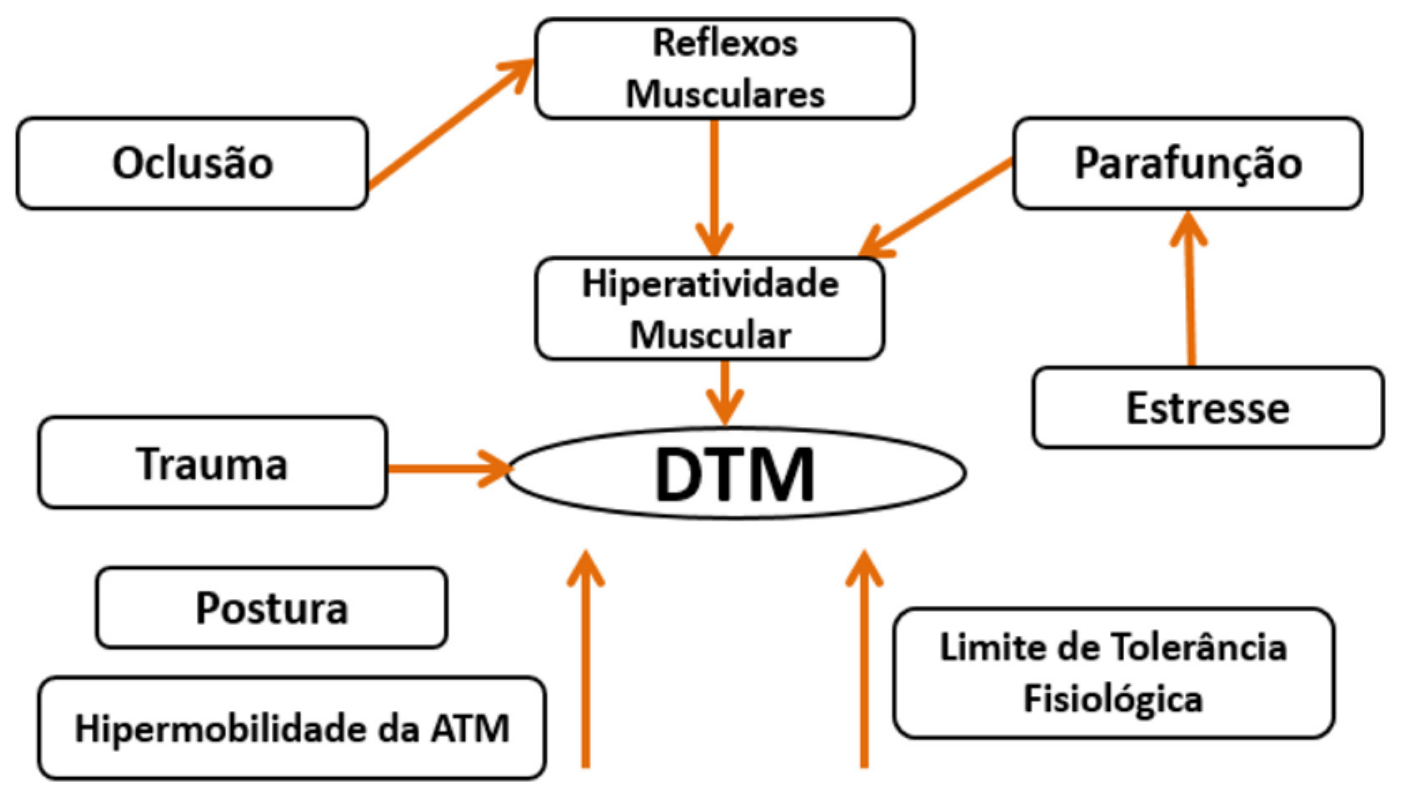

Figura 1. Etiologia da DTM, conforme PAIVA et al. (2007).

\section{Índices Diagnósticos}

Os índices encontrados na literatura são classificados como anamnésicos ou clínicos, esses são caracterizados pelo agrupamento operacional de conjuntos e sinais e ou sintomas sob a forma de itens e subitens. Para cada um é determinada uma pontuação ou um escore que no final são somados e permitem classificar os voluntários em subcategorias funcionais ou de severidade ${ }^{21}$.

Os questionários são utilizados com muita frequência para a avaliação de sintomas de DTM e podem ser aplicados de várias maneiras: em entrevista pessoal, com o auxílio de um entrevistador ou não, (auto-administráveis) e por telefone ${ }^{21}$.

Helkimo $^{22}$ foi um dos primeiros a criar um índice para a avaliação da DTM. . O Índice de Disfunção Clínica Craniomandibular - IDCCM (Clinical Dysfunction Index, ou Índice de Helkimo) tem por objetivo classificar os voluntários em categorias de severidade de sinais clínicos de DTM. É subdividido em cinco itens: limitação na amplitude de movimento mandibular, limitação na função da ATM, dor muscular, dor na ATM e dor no movimento mandibular. Para cada item são possíveis três pontuações, dependendo da avaliação clínica: 0, 1 e 5. Ao final, 
a somatória das pontuações de cada item permite classificar os voluntários em quatro categorias: sem sintomas de DTM (0 ponto), sintomas leves ( 1 a 4 pontos), moderados (5 a 9 pontos) e severos (10 a 25 pontos). Os dados necessários para preencher o item "a" do índice clínico devem ser obtidos pelo Índice de Mobilidade Mandibular (IMM) ${ }^{21}$.

O Questionário anamnésico de Fonseca et al. $^{23}$ foi elaborado nos moldes do Índice anamnésico de Helkimo, muito utilizado em estudos brasileiros sobre a disfunção, foi previamente testado em 100 pacientes com queixas compatíveis com DTM, tais participantes responderam o questionário e foram avaliados clinicamente pelo índice de Helkimo. Assim demonstrou uma confiabilidade de $95 \%$ e boa correlação com o índice clínico de Helkimo ${ }^{21}$. 0 questionário de Fonseca consiste em um formulário com 10 perguntas que abrange a condição física e emocional do indivíduo. Para cada pergunta, as respostas possíveis são "sim", "às vezes" ou "não", que são atribuídos os valores "10", "5" e "0", respectivamente. Para a análise global do questionário, todas as respostas são somadas, promovendo o diagnóstico em "sem disfunção", "com disfunção", com disfunção moderada "e" com disfunção grave "(varia de 0-15, 20-40, 45-65 e 70-100, respectivamente $)^{24}$.

O questionário anamnésico de Conti et al. $^{25}$ é composto por 10 perguntas, tendo três opções de resposta, foram oferecidas para: "sim", "não" ou "às vezes". O valor "2" foi atribuído a cada resposta indicando a presença do sintoma; Valor "0", por sua ausência; E valor "1", para a resposta "às vezes". A soma total dos valores obtidos permite a classificação da amostra em relação à $T M D$, como índice para mensurar a DTM. Os valores de 0 a 3, livres de DTM; valores de 4 a 8 , DTM leve; valores de 9 a 14, DTM moderada; valores de 15 a 23, DTM severa. Neste estudo os achados em estudantes na cidade de Bauru-SP foram que 34,84\% apresentavam sintomas leves e a ocorrência da disfunção foi prevalente em mulheres, aqueles com níveis de sintomas graves e moderados necessitavam de tratamento. A tensão emocional e os hábitos parafuncionais demonstraram fortes associações com DTM e a oclusão pareceu não influenciar na presença ou gravidade da DTM.
Segundo Chaves et al. ${ }^{26}$ o RDC/TMD é um índice diagnóstico bastante utilizado e aceito. Já foi traduzido em diversas línguas, inclusive para o português, na qual a parte intitulada como Eixo II do questionário foi aplicada em uma amostra de brasileiros, portanto, sua validação já foi realizada. O RDC/TMD tem caráter biaxial, permitindo mensurar de forma confiável os achados físicos no Eixo I e aliar o status psicossocial que envolve indivíduos com perfil de disfunção dolorosa crônica no Eixo I. Dessa forma, o RDC/TMD é uma das poucas ferramentas existentes na literatura que pode mesurar a DTM e as condições psicossociais relacionadas.

Porém são poucos os estudos que avaliam o impacto das condições dolorosas da DTM sobre a qualidade de vida das pessoas afetadas, em comparação aos estudos sobre diagnóstico, sinais e sintomas da DTM.

\section{Qualidade de vida}

A Organização Mundial de Saúde (OMS) estabeleceu que qualidade de vida é a percepção de uma pessoa na sua posição de vida em quanto ao contexto cultural e dos valores no local em que vive, em relação aos seus ideais, expectativas e níveis de preocupação ${ }^{27}$.

Os indicadores da saúde bucal que mais influenciam na qualidade de vida ( $Q V$ ) são as dores faciais e as dentárias que causam prejuízos ao sono e ao sistema estomatognático. A dor orofacial produz efeito negativo na qualidade de vida mais do que outras condições sistêmicas como diabetes, hipertensão ou úlcera ${ }^{27}$.

A DTM apresenta uma variedade de sinais e sintomas, onde os pacientes portadores possuem um elevado grau de comprometimento físico e mental, e exibem características clínicas comum com os portadores de outros tipos de doenças crônicas, refletindo negativo na qualidade de vida $(Q V)^{28,27}$. O conceito de $Q V$ relacionada com a saúde oral oferece grande oportunidade para sintetizar uma variedade de possíveis impactos psicossociais em relação a determinadas doenças bucais, sendo possível caracterizar a carga psicossocial da DTM e comparar esse impacto entre os seus diagnósticos específicos ${ }^{28}$. 
Entre as variáveis de qualidade de vida que foram estudadas por Biasotto-Gonzalez et al. ${ }^{14}$, as condições emocionais mostram-se alteradas quando se agregam a uma pior qualidade de vida em pacientes que sofrem com DTM. A associação entre o declínio da qualidade de vida e o estresse emocional é vista em vários estudos, especialmente nos estudos que utilizam tratamentos terapêuticos que promovem $\circ$ relaxamento, e melhoram a qualidade de vida.

Há diversos instrumentos para avaliar a qualidade de vida de pacientes com a disfunção temporomandibular em questão nesta revisão, como - Questionário de McGrill que é relatado por vários estudos como indicado para esta avaliação em pacientes com dor orofacial ${ }^{29}$.

O questionário de dor de McGill (MPQ) tem sido bastante utilizado para o impacto da dor na qualidade de vida do paciente com DTM, no qual utiliza-se questões relacionadas ao prejuízo social, desenvolvimento das atividades da vida diária e percepção do indivíduo paciente sobre a reação de terceiros à sua condição dolorosa ${ }^{30}$.

Uma tradução do questionário de McGill (MPQ) para a língua portuguesa ( $B r-M P Q)$ acrescentou algumas questões específicas, como: (1) prejuízo social; (2) atividades da vida diária; (3) percepção do outro; (4) tolerância à dor; (5) sensação de estar doente; (6) sensação de utilidade; (7) satisfação com a vida ${ }^{30}$.

Oral Health Impact Profile (OHIP) é uma escala avaliativa da saúde oral relatada para mensurar a qualidade de vida desenvolvido na Austrália, aplicado também a indivíduos com a disfunção, os resultados obtidos por este questionário indicam diferentes categorias de diagnóstico com ênfase psicossocial ${ }^{31}$. Porém, vários estudos tem utilizado apenas um fragmento deste questionário ० OHIP-1 4 32. Uma outra versão deste questionário é a OHIP49 que realiza a avaliação de qualidade de vida em pacientes que irão ser reabilitados com prótese dentária ${ }^{33}$.

O Medical Outcome Study 36 é um instrumento de medida de qualidade de vida desenvolvido no final dos anos 80 nos EUA. Aplicado em diversas condições com boa sensibilidade, eliminando-se o problema de distribuição excessiva das pontas de escala como excelente e muito ruim. Este instrumento foi traduzido e validado no Brasil para avaliar a qualidade de vida em pacientes com artrite reumatoide e mostrou-se adequado às condições socioeconômicas e culturais da população brasileira, devido a tal tem sido aplicado a estudos em pacientes com DTM $^{34}$.

Fleck et al. ${ }^{35}$ relata a necessidade de instrumentos de rápida aplicação, assim o Grupo de Qualidade de Vida da Organização Mundial de Saúde desenvolveu a versão abreviada do WHOQOL-100, - WHOQOL-bref. Este instrumento apresenta 26 questões divididas em quatro domínios: físico, psicológico, relações sociais e meio ambiente, tendo o mesmo sido traduzido para o português. A versão abreviada em português do WHOQOL-bref mostra que $\circ$ instrumento apresenta características satisfatórias de consistência interna, validade discriminante, validade de critério, validade concorrente e fidedignidade teste-reteste.

A Escala de Satisfação com Vida foi usada para avaliar a satisfação com a vida. Ele consistiu em cinco itens, cada um classificado em uma escala de 7 pontos de 1 ("Totalmente em desacordo") para 7 ("Concordo plenamente"). Foi calculado adicionando a pontuação de todos os itens, produzindo um possível índice de satisfação com a vida dentro do intervalo de 5 a 35 . As pontuações mais altas indicaram maior satisfação com a vida ${ }^{36-38}$. 
Quadro 2. Índices para mensurar a Qualidade de Vida e a descrição dos mesmos.

\begin{tabular}{|c|l|}
\hline ÍNDICES & \multicolumn{1}{c|}{ DESCRIÇÃO } \\
\hline Br-MPQ & $\begin{array}{l}\text { Categorias: prejuízo social, atividades da vida diária e percepção do outro, e } \\
\text { nas questões sobre tolerância à dor, sensação de estar doente, sensação de } \\
\text { utilidade e satisfação com a vida }{ }^{39} \text {. }\end{array}$ \\
\hline SF-36 & $\begin{array}{l}\text { Categorias: Avaliação geral da saúde, prejuízo social por consequência da } \\
\text { saúde física, prejuizo social por consequência de Problema emocional, nas } \\
\text { questões sobre tolerância a dor a duração, no que interferiu e quanto ao } \\
\text { comportamento } 40\end{array}$ \\
\hline OHIP-14 & $\begin{array}{l}\text { Categorias: Dor, paladar, dor persistente, refeições, desconforto para comer, } \\
\text { tensão e irritabilidade }{ }^{39} \text {. }\end{array}$ \\
\hline OHIP-49 & $\begin{array}{l}\text { Categorias: Atividade da vida diária, na questão sobre a tolerância a dor, a } \\
\text { localização, percepção do outro, tensão e desconforto, paladar, mudanças de } \\
\text { hábitos, prejuizo social, avaliação psicossocial }{ }^{33} \text {. }\end{array}$ \\
\hline WHOQOL-bref & Categorias: Físico, psicológico, social e meio ambiente. ${ }^{15}$. \\
\hline $\begin{array}{c}\text { Satisfaction with Life } \\
\text { Scale }\end{array}$ & Categoria: Vida pessoal. Satisfação de vida ${ }^{41}$. \\
\hline
\end{tabular}

A literatura apresenta muitas vezes a associação de mais de um instrumento, por exemplo Oral HealTH Impact Profile (OHIP-14) e Br-MPQ, para avaliar a qualidade de vida de pacientes com a DTM, talvez esta associação ocorra devido as variáveis contempladas em diferentes questionários auxiliarem na compreensão da condição de vida destes pacientes ${ }^{39}$.

\section{Discussão}

Um estudo avaliou 33 mulheres entre 20 e 40 anos quanto a severidade de DTM, saúde oral e qualidade de vida. Assim tal estudo utilizou o instrumento de Conti et al. ${ }^{25}$ validado na literatura para avaliar a severidade da DTM. Os achados indicaram elevada prevalência para indivíduos com uma disfunção $76 \%$, sendo que com nível médio foram os mais frequentes em relação aos demais níveis para a patologia, já dentre os critérios de qualidade de vida todos apresentaram uma relação estatisticamente significativa, com exceção de limitação física e funcional, um fator importante é que a limitação social e domínio socioemocional apresentou uma correlação positiva para a presença de DTM $^{32}$.

Uma população alemã diagnosticada com DTM pelo instrumento RDC/DC, observou que o distúrbio do paladar é prevalente nos pacientes com a disfução do que indivíduos sem a patologia, estando correlacionada a gradação da dor. Tais distúrbios sensoriais estão presentes em pacientes com dor crônica ${ }^{42}$.

Castanharo $^{43}$ verificou que a associação DTM e cefaléia primária representa forte impacto na qualidade de vida dos indivíduos, e que as cefaléias crônicas afetam mais significantemente o paciente.

Calderon et al. ${ }^{44}$ observou que a qualidade de vida em mulheres com DTM há mais de 6 meses, por meio do diagnóstico do RDC/DC, apresentaram uma pontuação média do OHIP de 1 1,72 e 11,74 para os grupos com e sem zumbido, respectivamente. Porém, a presença do zumbido não mostrou interferência na qualidade de vida destas, enquanto que a presença da dor sim, pois promove a incapaciente do indivíduo mais que os sintomas otológicos desta disfunção.

Tjakkes et al. ${ }^{45}$ observou em uma população de homens (05) e mulheres (90), atendidos em um serviço universitário, em que todos os pacientes apresentavam a DTM, assim utilizou-se o instrumento RDC/DC para promover o diagnóstico da mesma e a influencia na qualidade de vida por meio do instrumento SF-36. Os resultados apresentaram prevalência de $35,2 \%$ para pacientes que apresentaram artrose, osteoartrite e osteoartrose, ou seja, alterações articulares superaram as alterações musculares. Assim pacientes que apresentam mais de um ano com a disfunção verifica-se problemas físicos e limitação das atividades o que promove um impacto social, e muitas vezes o tratamento inicial não alivia a dor, tendo a qualidade de vida afetada. 
Costa et al..$^{33}$, em um estudo piloto, avaliou a qualidade de vida de pacientes edêntulos por meio do questionário Oral HealTH Impact Profile (OHIP-49), bem como a sensibilidade de músculos mastigatórios e alteração da dimensão vertical. Sendo assim, observou-se que não houve correlação entre variáveis protéticas e sensibilidade dos músculos mastigatórios, no entanto a qualidade de vida destes pacientes é afetada pela sintomatologia muscular. Porém, este estudo foi uma pesquisa piloto com um número amostral reduzido e não realizou a aplicação de um questionário para diagnóstico da DTM.

Nos estudos de Freitas et al. ${ }^{39}$ avaliaram mulheres de 19 a 53 anos, sendo que o valor amostral deste estudo foi de 27 participantes, num serviço para tratamento da DTM, assim observou-se que os aspectos de dor se apresentou interferência em atividades como o sono (35\%) e acometimento quanto ao apetite $(30 \%)$, trazendo prejuízos para a qualidade de vida, interferindo nas atividades diárias.

Estudo para avaliar a qualidade de vida em pacientes com disfunção temporomandibular que apresentavam distúrbios psicológicos, utilizou o Índice de Fonseca para realizar a triagem entre pacientes, em seguida aplicou-se o RDC/TMD Eixo I para mensurar alterações musculares nestes pacientes. Já para observar a condição de qualidade de vida aplicou-se o instrumento "WHOQOL-bref". Quanto aos resultados, foi encontrada uma associação entre distúrbios psiquiátricos e gravidade da DTM, o item "desejo de morte" esteve relacionado à DTM muscular grave. Para qualidade de vida, uma associação foi encontrada entre deslocamento de disco com redução e o domínio social; já os domínios físicos foram associados com DTM muscular ${ }^{15}$.

Oliveira et al. ${ }^{46}$ observou que de acordo com o questionário da Fonseca, a presença de DTM esteve associada à ansiedade; já a gravidade da disfunção a classificação de do estado de ansiedade (leve, moderada, grave); assim a presença de DTM pode reduzir a qualidade do sono e a qualidade de vida do indivíduo afetado.

Pacientes com o diagnóstico de DTM em serviço de dor orofacial, verificou-se que a satisfação da vida apresenta interferências nos aspectos emociaionais, funcionais e social nos pacientes com a desordem ${ }^{41}$.

Amador $^{40}$ observou a presença da disfunção e a qualidade de vida em pacientes de serviços odontológicos, houve predominância de DTM moderada, por meio do Questionário de Fonseca. Quanto a qualidade de vida, os domínios físicos e emocionais apresentaram menores escores, ou seja, quanto maior a dor relatada pelo paciente menor $\circ$ valor do domínio SF-36, interferindo na qualidade de vida dos portadores de DTM.

Pacientes com o diagnóstico de DTM em serviço de dor orofacial, verificou-se que a satisfação da vida apresenta interferências nos aspectos emociaionais, funcionais e social nos pacientes com a desordem ${ }^{41}$. O Quadro 3 aprenta os estudos acima citados quanto a população e local do estudo, e instrumento utilizado para mensurar a qualidade de vida. 
Quadro 3. Autor (ano), população, local do estudo e instrumento para avaliar a qualidade de vida.

\begin{tabular}{|c|c|c|c|}
\hline $\begin{array}{l}\text { Autor } \\
\text { (ano) }\end{array}$ & População & Local do Estudo & $\begin{array}{l}\text { Instrumento de Avaliação } \\
\text { Qualidade de Vida }\end{array}$ \\
\hline $\begin{array}{l}\text { Nixdorf et al. } \\
(2009)\end{array}$ & $\begin{array}{l}\text { Mulheres com média } \\
\text { de idade de } 38 \text { anos }\end{array}$ & Alemanha & Oral HealTH Impact Profile (OHIP) \\
\hline $\begin{array}{l}\text { Pereira et al. } \\
(2009)\end{array}$ & $\begin{array}{c}\text { Mulheres } 20 \\
\text { a } 40 \text { anos }\end{array}$ & Brasil & Oral HealTH Impact Profile (OHIP-14) \\
\hline $\begin{array}{l}\text { Amador } \\
(2016)\end{array}$ & $\begin{array}{c}\text { Mulheres e Homens } \\
\text { com média de idade } \\
\text { de } 31 \text { anos }\end{array}$ & Brasil & Short Form (SF-36) \\
\hline $\begin{array}{l}\text { Castanharo } \\
\text { (2011) }\end{array}$ & $\begin{array}{c}\text { Mulheres e Homens } \\
18 \text { a } 76 \text { anos }\end{array}$ & Brasil & Short Form (SF-36) \\
\hline $\begin{array}{l}\text { Calderon et } \\
\text { al. (2012) }\end{array}$ & $\begin{array}{l}\text { Mulheres com média } \\
\text { de idade de } 35 \text { anos }\end{array}$ & Brasil & Oral HealTH Impact Profile (OHIP-14) \\
\hline $\begin{array}{l}\text { Resende et al. } \\
\text { (2013) }\end{array}$ & $\begin{array}{c}\text { Mulheres e Homens } \\
\text { com média de idade } \\
\text { de } 36 \text { anos }\end{array}$ & Brasil & "WHOQOL-bref" \\
\hline $\begin{array}{c}\text { Costa et al. } \\
(2015)\end{array}$ & $\begin{array}{l}\text { Mulheres e Homens } \\
\text { com média de } \\
\text { idade de } 70 \\
\text { anos }\end{array}$ & Brasil & Oral HealTH Impact Profile (OHIP-49) \\
\hline $\begin{array}{l}\text { Freitas et al. } \\
\text { (2015) }\end{array}$ & $\begin{array}{l}\text { Mulheres } 19 \\
\text { a } 53 \text { anos }\end{array}$ & Brasil & $\begin{array}{l}\text { Oral HealTH Impact Profile (OHIP-14) } \\
\qquad B r-M P Q\end{array}$ \\
\hline $\begin{array}{l}\text { Oliveira et al. } \\
(2015)\end{array}$ & $\begin{array}{l}\text { Mulheres } \\
\text { maiores de } \\
18 \text { anos }\end{array}$ & Brasil & Short Form (SF-36) \\
\hline $\begin{array}{c}\text { Tiakkes et al. } \\
(2010)\end{array}$ & $\begin{array}{c}\text { Mulheres e Homens } \\
\text { de } \\
17 \text { a } 69 \text { anos }\end{array}$ & $\begin{array}{l}\text { Países } \\
\text { Baixos }\end{array}$ & Short Form (SF-36) \\
\hline $\begin{array}{l}\text { Amador } \\
(2016)\end{array}$ & $\begin{array}{l}\text { Mulheres com média } \\
\text { de idade de } 31 \text { anos. }\end{array}$ & Brasil & $\begin{array}{l}\text { Short Form (SF-36) } \\
B r-M P Q\end{array}$ \\
\hline $\begin{array}{l}\text { Boggero et al. } \\
(2016)\end{array}$ & $\begin{array}{l}\text { Mulheres e Homens } \\
\text { com média de idade } \\
\text { de } 45 \text { anos }\end{array}$ & EUA & Satisfaction with Life Scale \\
\hline
\end{tabular}

Para compreender e gerenciar pacientes com disfunção temporomandibular é necessário entender a disfunção temporomandibular suas comorbidades, como também observar a qualidade de vida. As implicações desta disfunção são graves, principalmente em relação às condições crônicas, assim o uso de estratégias de gestão biopsicossocial para reduzir dor e melhorar a função é a abordagem que tem demonstrado sucesso para a maioria dos casos $^{47}$. Paceintes com dor crônica como relatado por todos os estudos apresentam interferência direta na qualidade de vida de destes paceintes, promovendo problemas sociais, de trabalho e convívio.

Dentre os índices para diagnóstico da DTM - Questionário Anamnésico de Fonseca é um dos poucos instrumentos disponíveis em língua portuguesa, que determina a presença e severidade da disfunção, assim tem sido o índice mais utilizados em estudos brasileiros pela facilidade de aplicação e simplicidade quanto aos resultados obtidos, favorecendo a aplicação em estudos epidemiológicos populacionais. ${ }^{26}$

Se faz necessário, estudos que apresente intervenções terapêuticas distintas para DTM e avalie o desempenho dos mesmos quanto a qualidade de vida dos pacientes submetidos a tal e no controle dos sinais e sintomas. Pois a qualidade de vida é um termômetro para a satisfação do paciente, bem como, devolver o indivíduo ativo para as atividades e convívio social.

Os variados questionários para mensurar a qualidade de vida faz com que seja difícil interpretar os dados, prinicipalemente porque são questionários idealizados em outros países para populações com culturas diferentes em relação ao Brasil, sendo este fator talvez uma limitação para a aplicação neste país. Mas independente da população e questionário pacientes com DTM 
apresentam interferências na qualidade de vida. Sendo que a dor crônica promove a incapacidade do indivíduo exercer as atividades diárias.

Parece que a associação de questionários para qualidade de vida é bem vido na avaliação de pacientes com DTM, devido um só instrumento não contemplar todas as categorias necessárias para verificar o impacto da disfunção na qualidade de vida.

\section{Considerações finais}

A disfunção temporomandibular conforme a literatura revisada apresenta impacto na qualidade de vida do indivíduo portador da disfunção, diversos são os questionários para mensurar o quanto a DTM interfere nas atividades diárias dos pacientes, no entanto a associação de instrumentos é favorável para avaliar diferentes categorias quanto a qualidade de vida dos pacientes com disfunção.

\section{Conflitos de interesses}

Nenhum conflito financeiro, legal ou político envolvendo terceiros (governo, empresas e fundações privadas, etc.) foi declarado para nenhum aspecto do trabalho submetido (incluindo mas não limitandose a subvenções e financiamentos, conselho consultivo, desenho de estudo, preparação de manuscrito, análise estatística, etc).

\section{Referências}

1. Menezes MS, Bussadori SK, Fernandes KPS, BiasottoGonzalez DA. Correlação entre cefaléia e disfunção temporomandibular. Fisioter Pesqui. 2008;15(2):183-187. doi: $10.1590 /$ S1809-29502008000200012

2. Ash MM, Ramfjord SP, Schmioseroer J. Oclusão. 2.ed. São Paulo: Santos; 2001.

3. Paiva HJ, Vieira AMF, Cavalcante HCC, Medeiros ME, Gondim NFR, Barbosa RAD. Generalidades em oclusão: I Hábitos parafuncionais - II Gomas de mascar: prevenção e oclusão - III Biomecânica do estalido da ATM. In: Paiva HJ, editor. Oclusão: noções e conceitos básicos. São Paulo: Santos; 1997. p. 239-276.
4. Okeson JP. Tratamento das desordens temporomandibulares e oclusão. 4.ed. São Paulo: Artes Médicas; 2000.

5. Pessoa $C P$, Barreto $M B$, Santos $L B$, Alves TDB, Oliveira MC, Martins AG. Instrumentos utilizados na avaliação do impacto da dor na qualidade de vida de pacientes com dor orofacial e disfunção temporomandibular. Rev Baiana Saúde Pública. 2007;31(2):267-93.

6. de Oliveira AS, Bermudez CC, de Souza RA, Souza CMF, Dias EM, Castro CES et al. Impacto da dor na vida de portadores de disfunção temporomandibular. J Appl Oral Sci. 2003;1 1(2):138-43. doi: 10.1590/S1678$\underline{77572003000200010}$

7. Moreno BGD, Maluf AS, Marques AP, Crivello Júnior O. Avaliação clínica e da qualidade de vida de indivíduos com disfunção temporomandibular. Braz J Phys Ther. 2009;13(3):210-214. doi: 10.1590/S1413$\underline{35552009005000028}$

8. Romero-Reyers M, Uyanik JM. Orofacial Pain Management: Current Perspectives. J Pain Res. 2017;7:99-115. doi: $\underline{10.2147 / J P R . S 37593}$

9. Tomaz-Morais JF, Lucena LBS, Mota IA, Pereira AKFTC, de Lucena BTL, de Castro RD et al. Temporomandibular disorder is more prevalent among patients with primary headaches in a tertiary outpatient clinic. Arq Neuropsiquiatr. 2015;73(1 1):913-917. doi: 10.1590/0004-282X20150145

10. Buescher JJ. Temporomandibular joint disorders. Am Fam Physician. 2007;76(10):1477-82.

11. Piozzi R, Lopes FC. Desordens temporomandibulares: aspectos clínicos e guia para odontologia e fisioterapia. J Bras Oclusão, ATM Dor Orofacial. 2002;2(5):43-7.

12. Tosato JP, Caria PHF. Prevalência de DTM em diferentes faixas etárias. RGO. 2006;54(3):21 1-224.

13. Ozan F, Polat S, Kara I, Kucuk D, Polat HB. Prevalence study of signs and symptoms of temporomandibular disorders in a Turkish population. J Contemp Dent Pract. 2007 May $1 ; 8(4): 35-42$.

14. Silveira AM, Feltrin PP, Zanetti RV, Mautoni MC. Prevalence of patients harboring temporomandibular disorders in an otorhinolaryngology department. Braz J Otorhinolaryngol. 2007;73(4):528-32.

15. Biasotto-Gonzalez DA, de Andrade DV, Gonzalez TO, Martins MD, Fernandes KPS, Correa JCF et al. Correlação entre disfunção temporomandibular, postura e qualidade de vida. Rev Bras Crescimento Desenvolv Hum.2008; 1 8(1):7986. 
16. Resende CMBM, Alves ACM, Coelho TL, Alchieri JC, Roncalli AG, Barbosa GAS. Qualidade de vida e saúde geral em pacientes com disfunção temporomandibular. Braz. oral res. $2013 ; 27(2): 116-121$.

17. Sproesser JG. Características das relações interoclusais em indivíduos com mastigação realizada preferencialmente sobre um dos lados e sintomas de disfunção temporomandibular. Jornal Brasileiro de Oclusão, ATM e Dor Orofacial. 2002;2(5):26-31.

18. Marklund S, Wänman A. Risk factors associated with incidence and persistence of signs and symptoms of temporomandibular disorders. Acta Odontol Scand. 2010;68(5):289-299. doi: $10.3109 / 00016357.2010 .494621$

19. Roda RP, Bagan JV, Fernandez JMD, Bazan SH, Soriano YJ. Review of temporomandibular joint pathology. Part I: classification, epidemiology and risk factors. Med Oral Patol Oral Cir Bucal. 2017;12(4):292-298.

20. Sanders C, Liegey-Dougall A, Haggard R, Buschang $P$, Karbowski $S$, Riggs $R$ et al. Temporomandibular disorder diagnostic groups affect outcomes independently of treatment in patients at risk for developing chronicity: A 2-year followup study. J Oral Facial Pain Headache. 2016;30(3):187-202. doi: $10.11607 /$ ofph.1613

21. Chaves TC, de Oliveira AS, Grossi DB. Principais instrumentos para avaliação da disfunção temporomandibular, parte I: índices e questionários; uma contribuição para a prática clínica e de pesquisa. Fisioter Pesqui. 2008; 15(1):92-100. doi: 10.1590/S1809$\underline{29502008000100015}$

22. Helkimo M. Studies on function and dysfunction of the masticatory system, II: index for anamnestic and clinical dysfunction and occlusal state. Sven Tandlak Tidskr. 1974;67(2):101-21.

23. da Fonseca DM, Bonfate $G$, do Valle AL, de Freitas SFT. Diagnóstico pela anamnese da disfunção craniomandibular. Rev Gaucha Odontol. 1994;42(1):23-8.

24. Pessoa CP, Barreto MB, Santos LB, Alves TDB, Oliveira MC, Martins $A G$. Instrumentos utilizados na avaliação do impacto da dor na qualidade de vida de pacientes com dor orofacial e disfunção temporomandibular. Rev Baiana de Saúde Pública. 2007;31(2):267-293.

25. Conti PC, Ferreira PM, Pegoraro LF, Conti JV, Salvador MC. A across-sectional study of prevalence and etiology of signs and symptoms of temporomandibular disorders in high school and university students. J Orofac Pain. 1996; 10(3):254-62.
26. Chaves TC, de Oliveira AS, Grossi DB. Principais instrumentos para avaliação da disfunção temporomandibular, parte II: critérios diagnósticos; uma contribuição para a prática clínica e de pesquisa. Fisioterapia e Pesquisa. 2008;15(1):101-6. doi: 10.1590/ S1809-29502008000100016

27. Kuroiwa DN, Marinelli JG, Rampani MS, de Oliveira W, Nivodemo D. Desordens temporomandibulares e dor orofacial: estudo da qualidade de vida medida pelo Medical Outcomes Study 36 - Item Short Form Health Survey. Rev Dor. 2011 ; 12(2):93-98. doi: 10.1590/S1806$\underline{00132011000200003}$

28. Lemos GA, Paulino MR, Forte FDS, Beltrao RTS, Batista AUD. Influence of temporomandibular disorder presence and severity on oral health-related quality of life. Rev dor. 2015;16(1):10-14. doi: 10.5935/1806-0013.20150003

29. Rolim TS, Fabri GMC, Nitrini R, Anghinah R, Teixeira MJ, Siqueira JTT et al. Evaluation Of Patients With Alzheimer's Disease Before And After Dental Treatment. Arq Neuropsiquiatr.2014;72(12):919-924. doi: 10.1590/0004$\underline{282 \times 20140140}$

30. Pessoa CP, Barreto MB, Santos LB, Alves TDB, Oliveira MC, Martins AG. Instrumentos utilizados na avaliação do impacto da dor na qualidade de vida de pacientes com dor orofacial e disfunção temporomandibular. Rev Baiana de Saúde Pública. 2007;31(2):267-293.

31. John MT, Reissmann DR, Schierz O, Wassell RW. Oral healthrelated quality of life in patients with temporomandibular disorders. J Orofac Pain. 2007;21(1):4654.

32. Pereira TC, Brasolotto AG, Conti PC, Berretin-Felix G. Temporomandibular Disorders, Voice And Oral Quality Of Life In Women. J Appl Oral Sci. 2009;17:50-6.

33. Costa YM, Porporatti AL, Hilgenberg-Sydney PB, Bonjardim LR, Conti PCR. Deep pain sensitivity is correlated with oralhealth-related quality of life but not with prosthetic factors in complete denture wearers. J Appl Oral Sci. 2015;23(6):555-61. doi: 10.1590/1678-775720150174

34. Pimenta FAP, Simil FF, Torres HOG, Amaral CFS, Rezende $\mathrm{CF}$, Coelho TO et al. Avaliação da qualidade de vida de aposentados com a utilização do questionário SF-36. Rev Assoc Med Bras. 2008;54(1):55-60.

35. Fleck MPA. O instrumento de avaliação de Qualidade de Vida da Organização Mundial de Saúde (WHOQOL-100): características e perspectivas. Ciênc Saúde Coletiva. 2000;5(1):33-38. doi: 10.1590/S141381232000000100004

36. Cronbach LJ. Coefficient alpha and the internal structure of tests. Psychometrika. 1951;16(3):297-334. 
37. Diener E, Emmons RA, Larsen RJ, Griffin S. The Satisfaction With Life Scale. J Pers Assess. 1985;49(1):71-85. doi: $10.1207 / \mathrm{s} 15327752$ ipa4901 13

38. Pavot W, Diener E, Colvin CR, Sandvik E. Further validation of the Satisfaction with Life Scale: Evidence for the cross-method convergence of well-being measures. J Pers Assess. $1991 ; 57(1): 149-161$.

39. Freitas WMTM, dos Santos AKF, Saliba EM, da Silva EAM. Avaliação Da Qualidade De Vida E Da Dor Em Indivíduos Com Disfunção Temporomandibular. Rev Pesquisa em Fisioterapia. 2015;5(3):210-217. doi: 10.17267/22382704rpf.v5i3.636

40. Amador VD. Influência da Dor na Qualidade de Vida dos Portadores de Disfunção Temporomandibula [trabalho de conclusão de curso]. Campina Grande: Universidade Estadual da Paraíba; 2016.

41. Boggero IA, Rojas-Ramirez MV, de Leeuw R, Carlson CR. Satisfaction with Life in Orofacial Pain Disorders: Associations and Theoretical Implications. J Oral Facial Pain Headache. 2016;30(2):99-106. doi: 10.11607/ofph.1526

42. Nixdorf DR, Jhon MT, Schirz O, Berieter DA, Hellekat G. Self-Reported Severity Of Taste Disturbances Correlates With Severity Of Tmd Pain. J Oral Rehabil. 2009;36(1 1):792-800. doi: $10.1111 /$ i.1365-2842.2009.01996.x

43. Castanharo MS. Estudo da Qualidade de Vida em Pacientes Com Disfunção Temporomandibular e Cefaleias Primarias [dissertação]. Araraquara: Faculdade de Odontologia de Araraquara, Universidade Estadual Paulista; 2011.

44. Calderon PS, Hilgenberg PB, Rossetti LMN, Laurenti JVEH, Conti PCR. Influence of tinnitus on pain severity and quality of life in patients with temporomandibular disorders. J Appl Oral Sci. 2012;20(2):170-3. doi: 10.1590/S167877572012000200008

45. Tiakkes GH, Reinders JJ, Tenvergert EM, Stegenga B. TMD Pain: The Effect On Health Related Quality Of Life And The Influence Of Pain Duration. Health Qual Life Outcomes. 2010;8:46. doi: $10.1186 / 1477-7525-8-46$

46. Oliveira KL, Almeida GA, Lelis ER, Tavares $M$, Fernandes Neto AJ. Temporomandibular disorder and anxiety, quality of sleep, and quality of life in nursing professionals. Braz Oral Res. 2015;29(1):1 -7. doi: 10.1590/1807-3107BOR-2015. vol29.0070

47. Conti PCR, Pinto-Fiamingui LMS, Cunha CO, Conti ACCF. Orofacial Pain And Temporomandibular Disorders - The Impact On Oral Health And Quality Of Life. Braz Oral Res. 2012 ;26(supl 1):120-3. 